\title{
Hydrodynamic Study of a Continuous Large Volume Sorting and Concentration Technology for Particles and Cells in Micrometre Range
}

\author{
Nhut Tran-Minh ${ }^{1}$, Frank Karlsen ${ }^{2}$, Bendik Fyhn Terjesen ${ }^{1}$ \\ ${ }^{1}$ Norwegian Institute of Food, Fisheries and Aquaculture Research \\ Postboks 210, NO-1431 As, Norway \\ ${ }^{2}$ University College of Southeast Norway \\ Raveien 215, NO-3184 Borre, Norway \\ Nhut.Tran@nofima.no
}

\begin{abstract}
This paper presents the design of continuous sorting and concentration unit that can be used for particles or cells in the large volume of sea, waste, or fresh water. With the optimized profile of ellipse in combination with array of shield-like micropillars, efficiency of continuous sorting and concentration unit was examined by theoretical methods including Finite Element Method (FEM). With software (COMSOL 5.2) for computational fluid dynamics (CFD), we simulated unit in a laminar flow regime. Numerical results illustrate that the high velocity region which can be obtained along two side of unit and the penetration channels.
\end{abstract}

Keywords: computational fluid dynamics, sorting, concentration, micropillar

\section{Introduction}

One of the main challenges for the environmental and food industry today is to be able to perform more advanced sorting and concentration of particles or cells in large volumes of sea, waste or fresh water. There do exist a lot of technology for performing cross-flow or dead end filtration. However, this technology is only using simple holes or undefined matrix channels. The new continuous separation and concentration unit in this paper is a completely new way treat water and in particular complex water. The purpose is to get rid of as much liquid as possible with removing any defined particle. The purpose is also to concentrate the largest particles first including continuous separation call the refining technology.

It is expected that the use of this continuous sorting and concentration technology will open the door for treatment of many environmental and health problems and make it possible to analyse all biological activity with a defined geographical area.

\section{Material and Methods}

\subsection{Design of a Continuous Large Sorting and Concentration Unit}

The term shield-like micropillar is an element having the shape of a shield. As shown in Fig.1, shield-like micropillars are arranged on the contour of ellipse with the left half major axis shorter than right half major axis. These micropillars are together with a big blocking head to form the continuous large sorting and concentration unit. When the fluid flows through the unit, it faces the blocking head and is divided around the elliptical profile. The optimized elliptical will profile provides the largest high velocity region along the two sides of the ellipse [1]. This high velocity region aims to prevent the particle clogging at the penetrated channels. The main flow of fluid is able to push any large particles which are tending to block the entrance of the penetration channels. In other hand, the particles which is smaller than the gap between two adjacent micropillars will enter the penetration channels to the hole at the centre. 


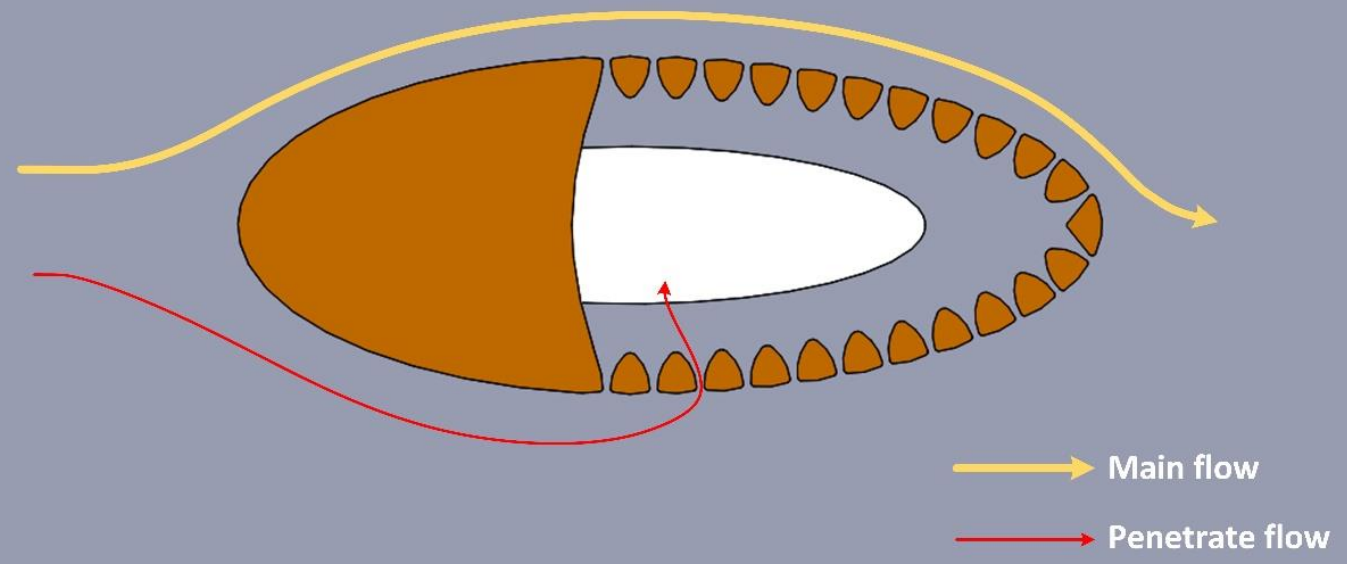

Fig. 1: Schematic of working principle of the continuous large sorting and concentration unit with shield-like micropillars.

For an incompressible fluid, equation of continuity (mass conservation of fluid) is defined as [2]

$$
A_{1} u_{1}=A_{2} u_{2}
$$

where $A_{1}, A_{2}$ are the cross-sectional area of the inlet and outlet of the penetration channels, respectively. $u_{1}$ and $u_{2}$ are fluid velocities at the cross-sectional area $A_{1}, A_{2}$, respectively. Since the width of inlet channel is less than the width of outlet channel, the fluid velocity at the inlet is always higher than fluid velocity at the outlet. (see Fig. 2). The gap between two adjacent micropillars in this paper is set to $20 \mu \mathrm{m}$ for the numerical simulation with COMSOL 5.2.

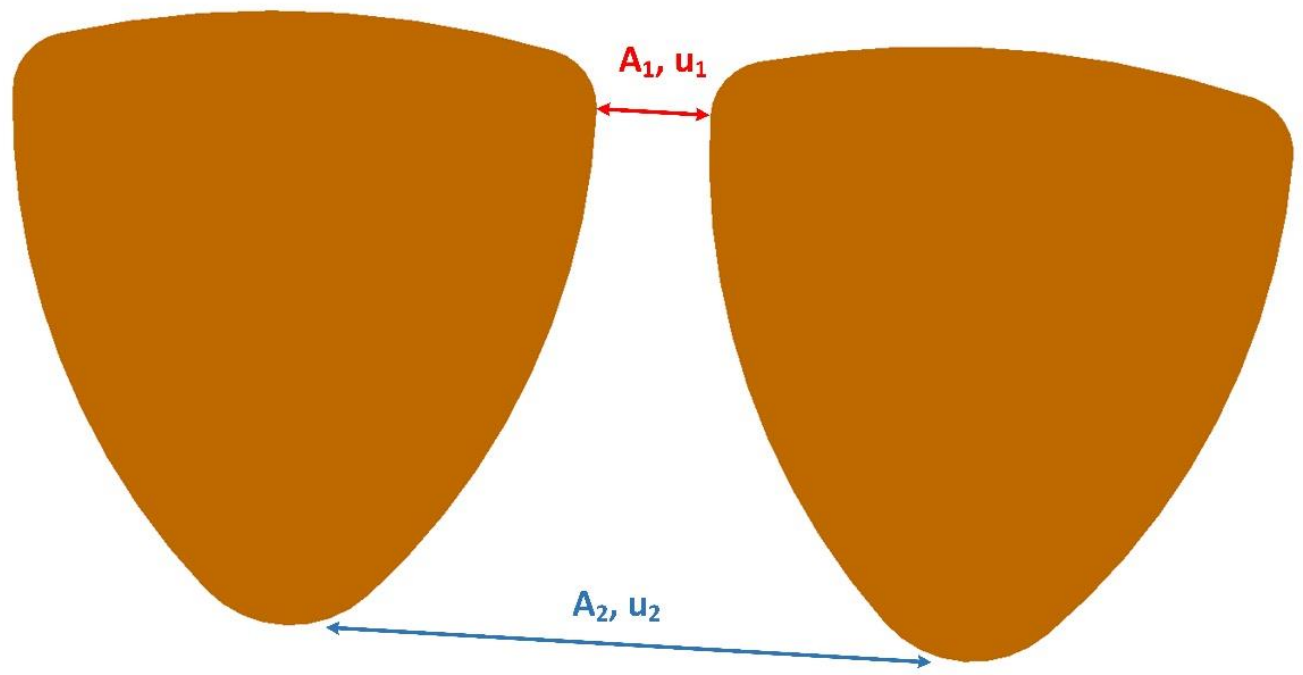

Fig. 2: Shield-like micropillars. 


\subsection{Modeling and Setting}

Multiphysics simulation software (COMSOL 5.2) was employed to evaluate the fluid performance of a continuous large sorting and concentration unit. During simulation, the incompressible steady flow condition was assumed. The physical properties of water were applied, the fluid density and the dynamic viscosity are $1000 \mathrm{~kg} . \mathrm{m}^{-3}$ and $8.9 \times 10^{-4} \mathrm{~Pa} . \mathrm{s}$, respectively. No-slip boundary condition is applied to the boundary on the wall. The velocity of $1 \mu \mathrm{m} / \mathrm{s}$ was set to the inlet boundary condition. The boundary condition for the outlet is zero pressure. A continuous large sorting and concentration unit was located at a distance of $6500 \mu \mathrm{m}$ from the inlet boundary condition. It is to make sure that the fluid will be fully developed when it reaches to the unit. The governing equations are solved with a complete mesh consists of 706324 domain elements, 103298 boundary elements, and 3919 edge elements.

\section{Results and Discussions}

Figure 3 illustrates the velocity field around the continuous sorting and concentration unit. The main flow is assumed to be from the left to right. The high velocity region marked with red colour is along two sides of elliptical profile. The low velocity region marked with dark blue colour appear at the front and rear of the unit. When both of small and large particles flow into the high velocity region, their velocities are accelerated dramatically. Since the large particles push each other at the entrance of penetration channel to prevent the phenomenon of clogging, the smaller particles are again accelerated to pass through penetration channel to the hole at the centre. (See Fig. 4 and Fig. 5).

The larger high velocity region is, the more fluid containing smaller particles go through array of shield-like micropillars. This means that we are able to collect or separate the large particles from a diluted solution without using any chemical or external physical source like magnetic field. While the large particles will follow the main flow stream and collected at the main outlet channel, the smaller particles follow the penetrated flow down to the lower layer and collected at the lower outlet channel.

As shown in Figure 5a, there are only few penetration channels closed to high velocity region of elliptical contour have an ability to accelerated dramatically. This can be improved with the optimization of elliptical profile.

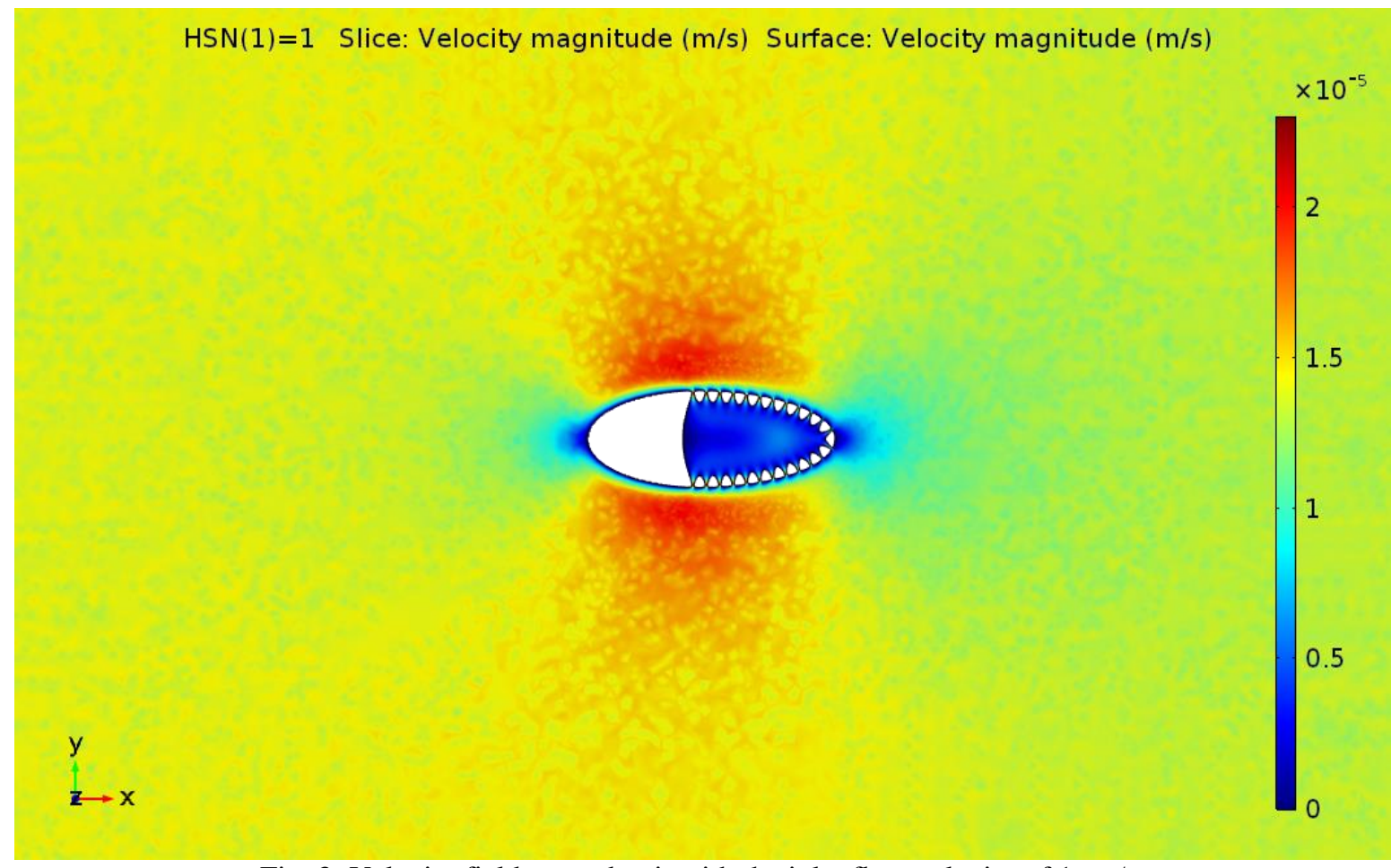

Fig. 3: Velocity field around unit with the inlet flow velocity of $1 \mu \mathrm{m} / \mathrm{s}$. 


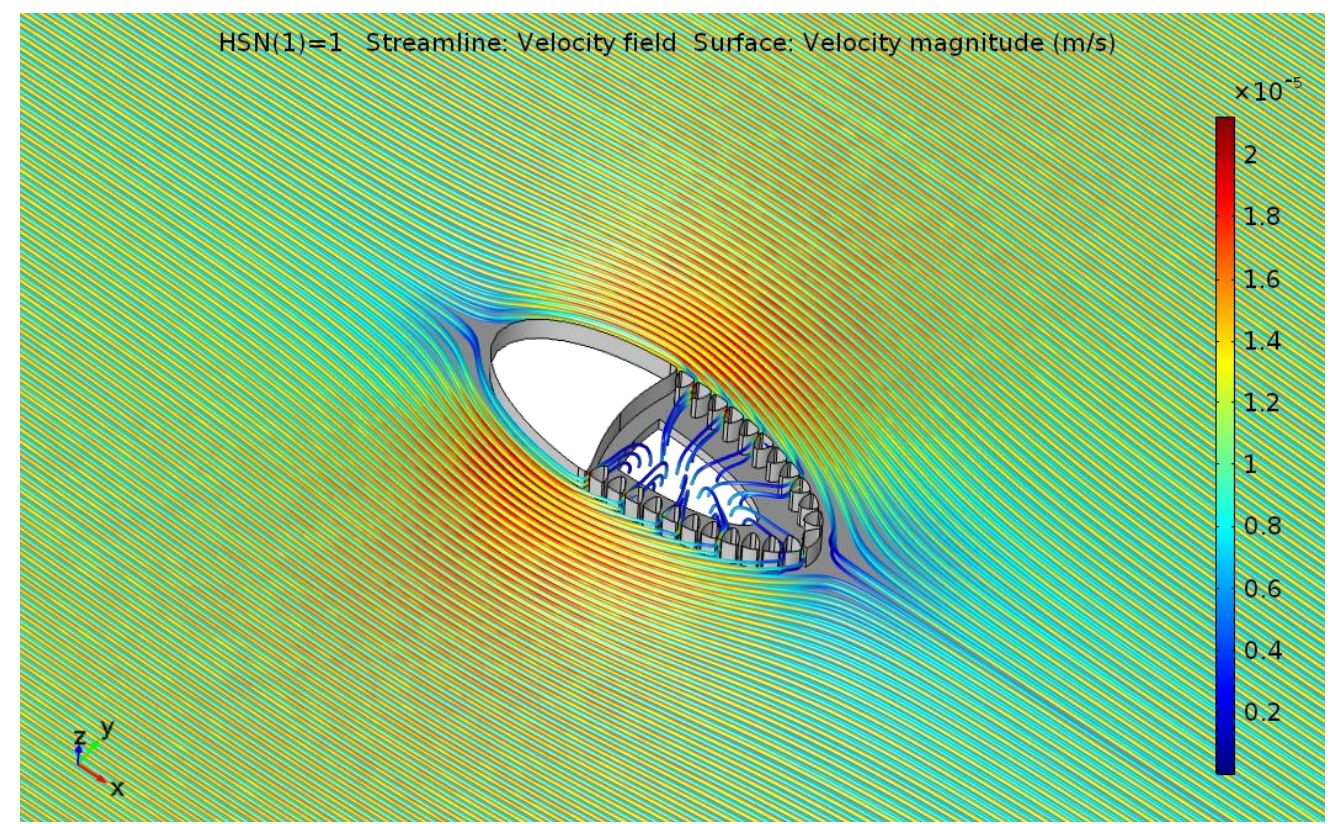

Fig. 4: Streamline flow visualization around unit with the inlet flow velocity of $1 \mu \mathrm{m} / \mathrm{s}$.

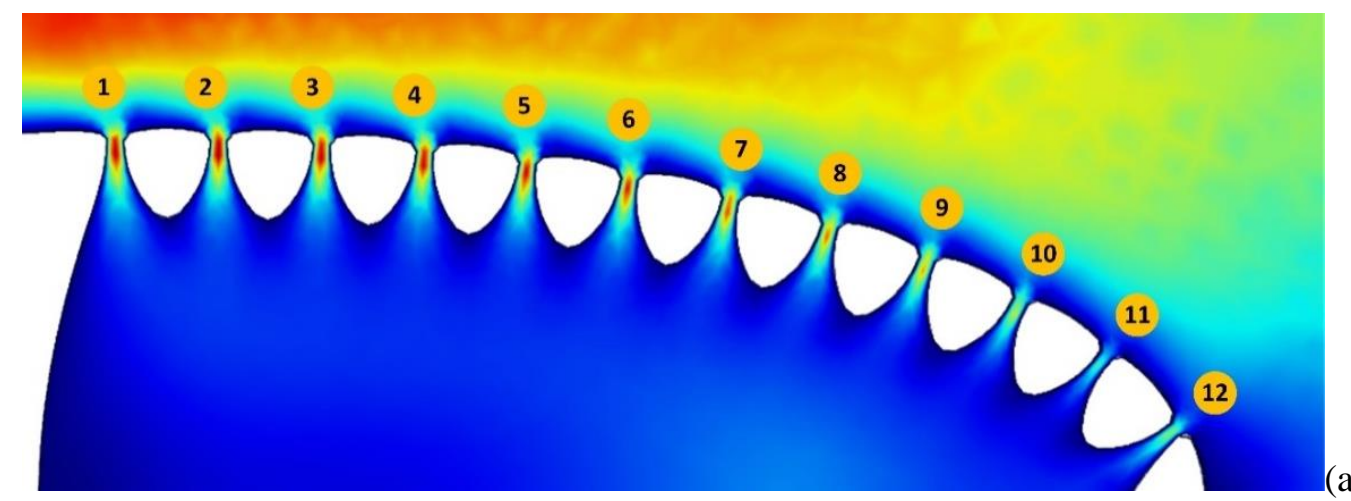

(a)

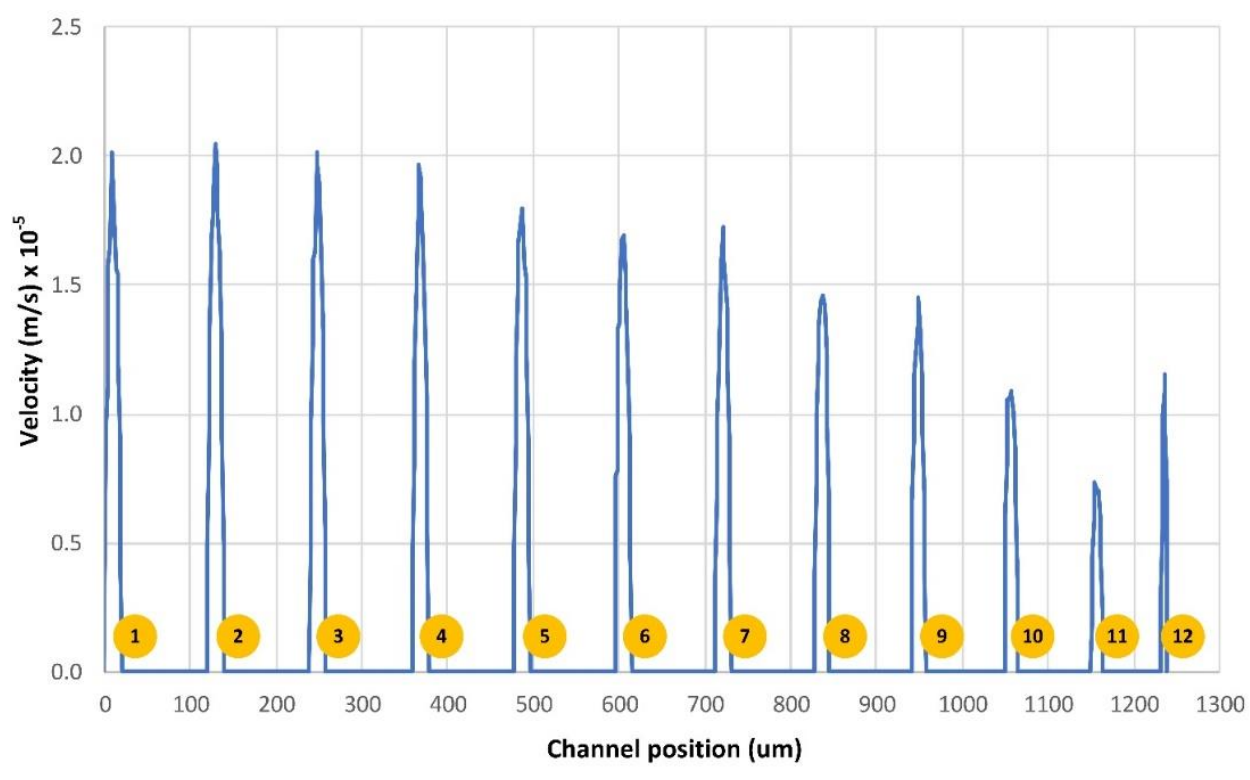

(b)

Fig. 5: The flow field simulation results. (a) The velocity field distribution. (b) cross-sectional velocity at penetration channels. 


\section{Conclusion}

In this paper, a continuous sorting and concentration unit which can be used for particles or cells in the large volume of sea, waste, or fresh water has been surveyed. With the optimized elliptical profile in combination with array of shieldlike micropillars, a continuous sorting and concentration unit was proposed with no-clogging, increased capacity and increased efficiency. The flow characteristics of unit was investigated by computational fluid dynamics. As future work, more complex fluidics such as human blood can be used for numerical study. Some practical experiments may be conducted in order to certify or validate the results from computational fluid dynamics.

\section{Acknowledgements}

The Research Council of Norway is acknowledged for the support through the Personal Post-doctoral Research Fellowships (HAVBRUK 2) [grant number 254862]. Partial funding for this work was obtained from the Norwegian PhD

Network on Nanotechnology for Microsystems, which is sponsored by the Research Council of Norway, Division for Science, under contract no. 221860/F40

\section{References}

[1] N. Tran-Minh, T. Dong, Q. Su, Z. Yang, H. Jakobsen, F. Karlsen, "Design and optimization of non-clogging counter-flow microconcentrator for enriching epidermoid cervical carcinoma cells," Biomedical Microdevices, vol. 13, no. 1, pp 179-190, 2011.

[2] I. G. Currie, Fundamental mechanics of fluids. McGraw-Hill, Inc, New York, 1993. 